\title{
Patterns of Using Complementary and Alternative Medicine by Stroke Patients at Two University Hospitals in Korea
}

\author{
Yong-II Shin ${ }^{1,2}$, Chung-Yong Yang ${ }^{1,2}$, Min-Cheol Joo ${ }^{1,2}$, Sam-Gyu Lee ${ }^{3}$, Jae-Hyung Kim ${ }^{3}$ \\ and Myeong Soo Lee Le $^{4,5}$
}

${ }^{1}$ Department of Physical Medicine \& Rehabilitation, ${ }^{2}$ Institute of Wonkwang Medical Science, Wonkwang University School of Medicine, Iksan, Korea, ${ }^{3}$ Department of Physical Medicine \& Rehabilitation, Research Institute of Medical Science, Chonnam National University Medical School \& Hospital, Gwangju and ${ }^{4}$ Center for Integrative Medicine, Institute of Medical Science, Wonkwang University, Iksan, Korea

\begin{abstract}
This study measured the prevalence of complementary and alternative medicine (CAM) use among Korean stroke patients. Questionnaire-based 20-min interviews were conducted at the hospitals by a trained nurse after an outpatient visit. It included questions on demographic information, clinical information and the utilization of CAM. Of 304 stroke-patient respondents, $164(54 \%)$ had used CAM, of which $66 \%$ had started taking CAM products following suggestions from family members and other relatives. Of the $57 \%$ of users who felt that CAM was effective, $84 \%$ considered that it improved the symptoms of stroke and $16 \%$ felt it was effective in achieving psychological relaxation. Of the eight CAM categories used by respondents, $92 \%$ used traditional Oriental medical treatments, $36 \%$ used plant- and animalderived over-the-counter health care products, $24 \%$ used minerals and vitamins, and $11 \%$ used manual therapies. The majority of stroke patients $(68 \%)$ were trying a new type of CAM, and half of the respondents $(45 \%)$ relied on the knowledge of their general practitioner about CAMs when deciding whether to use them. Most of the stroke patients in this study used CAM, and a half of them reported beneficial effects. Despite the presence of adverse side effects, they tended to be used without discussion with chief physicians, and hence physicians should be actively involved in the usage of CAM.
\end{abstract}

Keywords: complementary and alternative medicine (CAM) - stroke - survey

\section{Introduction}

Stroke is the third leading cause of mortality in the world (1). It is also second highest cause of mortality (13.9\%, 70.3 persons per 100000 in 2004) and morbidity in adults and the most common cause of disability in Korea (2). Currently, about $33 \%$ of disabilities occurring in subjects older than 35 years are caused by stroke in

\footnotetext{
${ }^{5}$ Present address: Complementary Medicine, Peninsula Medical School, Universities of Exeter \& Plymouth, Exeter, UK

For reprints and all correspondence: Myeong Soo Lee, $\mathrm{PhD}$,

Complementary Medicine, Peninsula Medical School, Universities of

Exeter \& Plymouth, 25 Victoria Park Road, Exeter, EX2 4NT, UK.

Tel: 44 (0)1392 439035; E-mail: drmslee@gmail.com;

myeong.lee@pms.ac.uk
}

Korea, with only $4-20 \%$ of them exhibiting complete functional recovery. Because of the chronic nature of this condition, patients were normally treated with both physical medicine and rehabilitation. A significant proportion of rehabilitation medicine patients use, and frequently perceive a benefit from, alternative medicine (3). The reasons that people choose such therapies include positive views of complementary therapies, limitation of conventional medicines, concern about the adverse effects of conventional medicine, communication with doctors, and the increased availability of complementary therapies (4-6).

Oriental medicine, which is considered one type of Complementary and alternative medicines (CAM) in 
Western medicine, largely presented by acupuncture, herbal medicine etc. and covered by public medical insurance had been a major form of health care for patients in Korea. Thus, other options for rehabilitation of patients with stroke are CAM in Korea.

In recent years, there has been growing interest and publications in CAM for various ranges of medical conditions. Few surveys that examine CAM use in patients with stroke are available $(7,8)$. Recognizing the increasing interest in CAM among the public, the aim of this survey was to assess stroke patients' expectations, prevalence of use, and perceived effectiveness of CAM.

\section{Methods}

\section{Study Design and Population}

Our data were collected through face-to-face interviews on the utilization of CAM among patients with stroke in Korea. The interviews were conducted from May 2004 to April 2005 in two hospitals in Iksan and Kwangju city. Only patients who (i) had sufficient mental ability to understand all questions on the questionnaire and (ii) had been diagnosed with stroke at least 1 month previously were included. Questionnaire-based interviews lasting $20 \mathrm{~min}$ were conducted at the hospitals by a trained nurse after an out-patient visit. The responders first answered demographic questions, followed by questions about any treatments that their physicians had not prescribed. Working diagnosis and duration of stroke were confirmed by reviewing medical records.

\section{Survey Instrument}

CAM is defined as any intervention not prescribed by physicians, which is not presently considered to be part of conventional medicine (9), and is divided into the following eight categories: (i) traditional Oriental medicine prescribed or treated by Korean traditional medical doctors such as herbs, herbal mixture and acupuncture, (ii) plant- and animal-derived over the counter health products, (iii) minerals and vitamins, (iv) manual therapies, (v) spiritual mind-body medicine, (vi) self prescribed traditional folk remedies, (vii) bio-electromagnetic therapies and (viii) other remedies such as oxygen generator and charcoal, which is believed to be good for removing toxicity and working as an oxygen generator. Each category is included on the basis of a preliminary survey on the most frequent forms of CAM use by Koreans (unpublished data). In Korea, traditional Oriental medicine was performed only by certified Korean medical doctors, so each category was separated from over the counter plant- and animal-derived health products.
Patients confirming that they had used CAM for stroke were asked for their perceptions of its efficacy, motivation for use, major symptoms leading to use, total cost, source of payment, adverse effects, source of information, total number of CAMs, types of CAM and intention to use new CAM. Adverse effects in CAM can be classified using four conventional categories pharmacologically predictable effects, idiosyncratic reactions, reactions developed during long-term therapy and delayed effects such as carcinogenicity and teratogenicity (10). Furthermore, this also identifies those effects that occur as a result of practitioners' insufficient medical competence (10).

\section{Data Analyses}

Statistical analyses were performed using Sigmastat for Windows version 3.1(Systat Software Inc., San Jose, CA, USA). Comparison between CAM user and non-users were made using the Mann-Whitney U test for continuous variables and the chi-squared test for categorical variables. The continuous data were not normally distributed and hence the data are presented as medians and interquartile ranges.

\section{Results}

\section{Characteristics of Survey Population}

Of the 331 patients interviewed, 304 of the questionnaires were completed (92\% response rate), 16 denied the interview and 12 quit during the process. The demographic characteristics of the survey respondents are summarized in Table 1. The CAM users had significantly

Table 1. Demographic data of the participants

\begin{tabular}{llllll}
\hline $\begin{array}{l}\text { Demographic } \\
\text { characteristics }\end{array}$ & Total & $\begin{array}{l}\text { CAM } \\
\text { user }\end{array}$ & Non-user & $t$ or $\chi^{2}$ & $P$ \\
\hline $\begin{array}{l}\text { All respondents } \\
\text { Age, median }\end{array}$ & 304 & 164 & 140 & & \\
$\quad$ (IRQs) & $65(56-72)$ & $65(56-72)$ & $64(57-71)$ & & 0.34 \\
Sex & & & & 5.30 & 0.02 \\
$\quad$ Male & $144(53 \%)$ & $92(56 \%)$ & $59(42 \%)$ & & \\
$\quad$ Female & $143(46 \%)$ & $72(44 \%)$ & $81(58 \%)$ & & \\
$\begin{array}{l}\text { Diagnosis } \\
\text { Infarction }\end{array}$ & $196(64 \%)$ & $129(79 \%)$ & $90(64 \%)$ & & \\
$\quad \begin{array}{l}\text { Haemorrhage } \\
\text { SAH }\end{array}$ & $58(20 \%)$ & $29(18 \%)$ & $35(25 \%)$ & & \\
$\begin{array}{c}\text { Duration of } \\
\text { stroke } \\
\text { (months), }\end{array}$ & $3(1-12)$ & $7(2-29)$ & $1(1-3)$ & & $0.01 \%$ \\
$\begin{array}{l}\text { medians, } \\
\text { IRQs }\end{array}$ & & $6(3 \%)$ & $15(11 \%)$ & & \\
\hline
\end{tabular}

SAH, subarachnoid hemorrhage; IRQs, interquartile ranges. 
Table 2. Perception of stroke patients on CAM usages

\begin{tabular}{|c|c|c|}
\hline & No. & $\%$ \\
\hline \multicolumn{3}{|l|}{ Discussion the use of CAM with their GP } \\
\hline Yes & 59 & 36 \\
\hline No & 105 & 64 \\
\hline \multicolumn{3}{|l|}{ Intention to use a new type of CAM } \\
\hline Yes & 111 & 68 \\
\hline No & 53 & 32 \\
\hline \multicolumn{3}{|c|}{ Reliability of knowledge of their GP about CAM } \\
\hline Yes & 76 & 46 \\
\hline No & 88 & 54 \\
\hline \multicolumn{3}{|l|}{ Motivation for using CAM } \\
\hline $\begin{array}{l}\text { Obscure expectation of CAM such as } \\
\text { complete recovery by several } \\
\text { times of treatments }\end{array}$ & 103 & 63 \\
\hline Trust of CAM & 31 & 19 \\
\hline Friend and Family request & 30 & 18 \\
\hline \multicolumn{3}{|l|}{ Major symptom leading to the use of $\mathrm{CAM}^{*}$} \\
\hline Motor weakness and paralysis & 96 & 59 \\
\hline Impairment of gait & 29 & 18 \\
\hline Impairment of activities of daily living & 21 & 13 \\
\hline Disability of urination and defection & 19 & 12 \\
\hline Spasticity & 17 & 10 \\
\hline Fatigue & 16 & 10 \\
\hline Chronic pain & 14 & 9 \\
\hline \multicolumn{3}{|l|}{ Source of information } \\
\hline Friends and Family & 108 & 66 \\
\hline Other patients & 26 & 16 \\
\hline Their own physicians & 15 & 9 \\
\hline Health practitioners & 11 & 7 \\
\hline Mass media and books & 4 & 2 \\
\hline \multicolumn{3}{|l|}{ Onset of use CAM } \\
\hline $0-3$ months & 122 & 74 \\
\hline 3-6months & 26 & 16 \\
\hline Over 6 months & 16 & 10 \\
\hline \multicolumn{3}{|l|}{ Duration of using CAM } \\
\hline $0-3$ months & 83 & 51 \\
\hline $3-6$ months & 60 & 36 \\
\hline Over 6 months & 21 & 13 \\
\hline
\end{tabular}

longer duration of illness (median, 7 months; IQR, 3-29 months), and 105 (63\%) of them said that they did not discuss the use of CAM with their general practitioner (GP) (Table 2). Infarction patients (79\%) and males $(56 \%)$ used CAM more than those with other types of stroke and females, respectively. One hundred and eleven $(68 \%)$ patients replied that they were trying a new type of CAM, while $53(32 \%)$ stated that they would not try CAM.

\section{Reasons and Motivations for CAM Use}

One hundred and sixty-four (54\%) subjects reported having used CAMs at the time of the survey. The majority of CAM users were motivated by obscure expectations of CAM such as complete recovery by several times of treatments $(63 \%)$, confidence in CAM $(19 \%)$ and requests by friends and family members $(18 \%)$. The major symptoms leading to the use of CAM were motor weakness and paralysis (59\%), impairment of gait $(18 \%)$, impairment of activities of daily living $(21 \%)$, impairment of urination and defection $(12 \%)$, spasticity $(10 \%)$ fatigue $(10 \%)$ and chronic pain $(9 \%)$. The most important sources of information regarding CAM were friends, family members and other relatives $(66 \%)$, other patients $(16 \%)$, their own physician $(9 \%)$ and other practitioners $(7 \%)$. Neither the mass media $(n=2,1 \%)$ nor books related to health care $(n=2,1 \%)$ were important sources of information. A total of $54 \%$ of the CAM users did not rely on the knowledge of their GP about CAMs. Most patients (74\%) used CAM within 3 months of the onset of stroke, with $51 \%$ of them using CAM for up to 3 months, $36 \%$ using CAM for 3-6 months and $13 \%$ using CAM for more than 6 months.

\section{Perceived Effectiveness and Adverse Effects of CAM}

Slightly more than half $(57 \%)$ of the users considered CAM to be effective, $43 \%$ reported it to be ineffective, with $15 \%$ reporting adverse side effects, of which $46 \%$ reported drug toxicity, $25 \%$ reported fatigue and weakness and $20 \%$ reported allergies and skin problems.

\section{Types and Prevalence of CAM Used and Cost of Them}

Of the eight CAM categories used by respondents, $92 \%$ used Oriental medicine, $36 \%$ used plant- and animalderived over the counter health products, $24 \%$ used minerals and vitamins, $11 \%$ used manual therapies, $8 \%$ used charcoal and oxygen generator, $7 \%$ used self prescribed traditional folk remedies, $6 \%$ used spiritual, mind, and body methods and $1 \%$ used bioelectromagnetic therapies (Fig. 1). Some of the responders used at least two CAMs to treat symptoms, and they spent a median of US\$ 373 (IQR, US\$ 105-1099) for the CAM. Most of them $(60 \%)$ paid for CAM themselves, with the remainder $(40 \%)$ paid for by medical insurance.

\section{Discussion}

This study demonstrates that a high proportion of stroke patients both use and frequently perceive a benefit from CAM therapies. Of those interviewed, $54 \%$ had used at least two CAM therapies, and $57 \%$ perceived the 


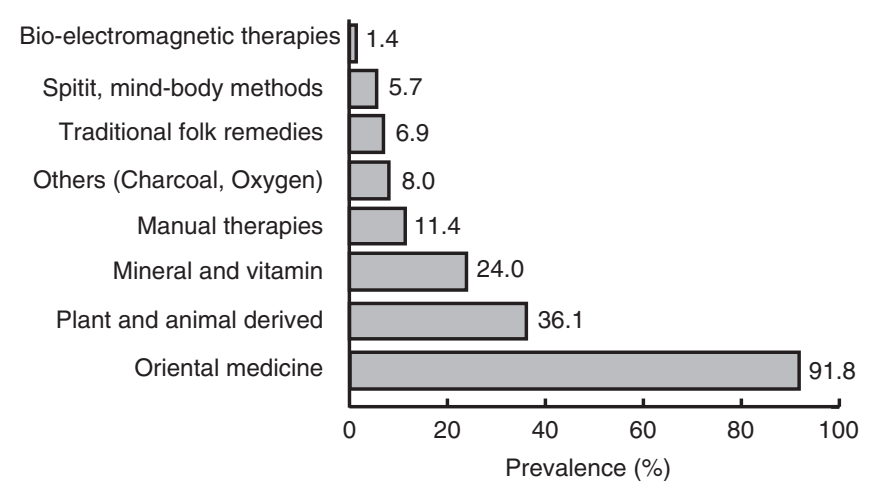

Figure 1. Type of CAM used by strokes patients (multiple statements are considered). Traditional folk remedies, self-prescribed remedies; Oriental medicine, herbs, herbal mixture and acupuncture prescribed or treated by Korean traditional medical doctors.

Table 3. Effectiveness of CAM and cost related information

\begin{tabular}{lcc}
\hline & No. & $\%$ \\
\hline Effectiveness of CAM & 78 & 48 \\
Effective for symptoms & 15 & 9 \\
Psychological effectiveness & 71 & 43 \\
No effectiveness & & \\
Adverse effects & 24 & 15 \\
Yes & 120 & 73 \\
No & 20 & 12 \\
Don't know & & 46 \\
Kind of adverse effects & 11 & 25 \\
Drug toxicity & 6 & 20 \\
Fatigue and weakness & 5 & 8 \\
Allergies and skin problems. & 2 & - \\
Worse than before & $3(2-5)$ & - \\
Total no of CAM used, median (IRQs) & $373(105-1099)$ & \\
Total cost (US\$), median (IRQs) & & 60 \\
Payment source & 98 & 40 \\
Burden by themselves & 66 & \\
Medical insurance &
\end{tabular}

CAM, complementary and alternative medicine; IRQs, interquartile ranges.

therapies used to be beneficial. Moreover, most patients $(68 \%)$ were using a new type of CAM. However, $64 \%$ of them did not discuss their physicians about the use of CAM.

The $54 \%$ prevalence of CAM use in this study is higher than the $26.5 \%$ use previously reported in 117 Saskatchewan stroke patients (7), the $36 \%$ use of CAM in 10572 cardiovascular disease cases excluding prayer (11), the $32.5 \%$ use of 252 patients with chronic heart failure (12) and the $18.5 \%$ use of 215 patients with neurological disorders (13). This result may echo the parallel medical systems of Western and Oriental Medicine in Korea (14).
Among the 164 current CAM patients (54\% of total respondents), most (92\%) used oriental medicine such as acupuncture, herbal remedies prescribed by Korean traditional medical doctors in combination with conventional medicines. The highest rate of CAM usage was among infarction patients, which may be due to them constituting most of the stroke victims. Moreover, a higher risk of recurrence and greater severity may prevent other types of stroke patients from using CAM.

The duration of diseases differed significantly between CAM users and non-users. A longer treatment period and overcoming the acute and subacute stages of diseases increased the chance of receiving information on the effectiveness of CAM from friends and relatives.

The most important sources of information and motivation to use CAM were obscure expectations about the effectiveness of CAM such as complete recovery by several times of treatments, confidence in CAM and the recommendations of family members and other relatives. Twenty-four patients reported adverse effects of CAM use in our study, including drug toxicity, fatigue, weakness and skin problems. These results show that stroke patients and their families should be educated about the efficacy and adverse effects of CAM.

Our results suggest that the use of CAM improves the physiological and psychological symptoms of stroke patients. Stroke patients may regard the use of CAM as a way to actively and independently contribute to the treatment of their disease.

The main symptoms leading to the use of CAM were motor weakness and paralysis, which is consistent with a previous report. CAM was first used within 3 months of the occurrence of stroke, and various types of CAM were used regularly. The median total cost was US\$ 373 (IQR, US\$ 105-1099), which is higher than that reported for other surveys (7). However, since medical insurance paid for $40 \%$ of the costs in Korea, the real cost may be twice this figure.

Many of the stroke patients $(68 \%)$ were trying a new type of CAM, and more than half of the users $(63 \%)$ were motivated by obscure expectations about the effectiveness of CAM. These results show that the expectations of CAM are higher than those of conventional therapies. Therefore, physicians should recognize and consider their patients' desire for improving their health state, and hence be able to advise patients on the use of CAM.

We found that oriental medicine $(92 \%)$ was the most widely used CAM for stroke, which may be attributable to acupuncture being considered as a basic treatment for stroke in Korea, especially infarction. In addition to this, CAM represents one of the main medical systems in Korea, and public insurance schemes support certain therapies that are included in categories of CAM. The usage of oriental medicine in arthritis was reported at $21 \%$, with each patient using an average of 
1.3 CAMs (15). Therefore, oriental medicine is used more by stroke patients than by patients with other diseases, including rheumatoid arthritis.

This study is subject to several potential limitations. The survey was conducted with a small number of patients in only two cities, and hence the results might not be generalizable to stroke patients in other regions or to Korea as a whole. In addition, our study did not investigate the objective effectiveness of CAM in stroke patients, for example by not showing patients who were not stable on their conventional therapy becoming stable when CAM was added. Instead, our patients only provided subjective answers about improvements in their symptoms. Therefore, future studies should recruit patients from diverse geographic regions, separate the types of stroke and obtain objective data on the efficacy of using CAM.

In conclusion, we found that many stroke patients used CAM therapies, with most patients using Oriental medicine but also with $64 \%$ of them not informing their physicians about their use of CAM. Family members, other relatives, and other patients represented significant sources of CAM information. Many stroke patients $(68 \%)$ were trying a new type of CAM, and half of respondents relied on the knowledge about CAM of their physicians. Doctors should therefore recognize that complementary medicines are widely used by stroke patients and should also appreciate that these medicines can cause adverse effects. Doctors should also determine what CAM their patients use, and provide education on the evidence-based efficacy and side effects of CAM to both patients and their families.

\section{Acknowledgements}

This paper was supported by Wonkwang University in 2005 .

\section{References}

1. World Health Orgarnization. Death from stroke. Accessed at: http://www.who.int/entity/cardiovascular_diseases/en/cvd_atlas_16_ death from stroke.pdf. (accessed 15 November 2006).

2. Korea National Statistical Office. Statistical survey of death causes in 2004. Seoul, Korea, 2005.

3. Wainapel SF, Thomas AD, Kahan BS. Use of alternative therapies by rehabilitation outpatients. Arch Phys Med Rehabil 1998;79:1003-5.

4. Furnham A, Kirkcaldy B. The health beliefs and behaviours of orthodox and complementary medicine clients. $\mathrm{Br} J$ Clin Psychol 1996;35:49-61.

5. Lee MS, Lee MS, Lim HJ, Moon SR. Survey of the use of complementary and alternative medicine among Korean diabetes mellitus patients. Pharmacoepidemiol Drug Saf 2004;13:167-71.

6. Vincent C, Furnham A. Why do patients turn to complementary medicine? An empirical study. Br J Clin Psychol 1996;35:37-48.

7. Blackmer J, Jefromova L. The use of alternative therapies in the Saskatchewan stroke rehabilitation population. BMC Complement Altern Med 2002;2:7.

8. Golomb MR, Hune S, MacGregor DL, deVeber GA. Alternative therapy use by Chinese-Canadian children with stroke and cerebrovascular disease. J Child Neurol 2003;18:714-7.

9. National Center for Complementary and Alternative Medicine. What is complementary and alternative medicine (CAM)? Accessed at: http://nccam.nih.gov/health/whatiscam/(accessed 15 November 2006).

10. Ernst E, Barnes J. Methodological approaches to investigating the safety of complementary medicine. Complement Ther Med 1998:6:115.

11. Yeh GY, Davis RB, Phillips RS. Use of complementary therapies in patients with cardiovascular disease. Am J Cardiol 2006; 98:673-80.

12. Zick SM, Blume A, Aaronson KD. The prevalence and pattern of complementary and alternative supplement use in individuals with chronic heart failure. J Card Fail 2005;11:586-9.

13. Ryan M, Johnson MS. Use of alternative medications in patients with neurologic disorders. Ann Pharmacother 2002; 36:1540-5.

14. Shim BS, Koh BH, Ahn KS. Education in Oriental Medicine in Kyung Hee University. Evid Based Complement Alternat Med 2004;1:331-34.

15. Kim HA, Seo YI. Use of complementary and alternative medicine by arthritis patients in a university hospital clinic serving rheumatology patients in Korea. Rheumatol Int 2003; 23:277-81.

Received July 27, 2006; accepted December 4, 2006 


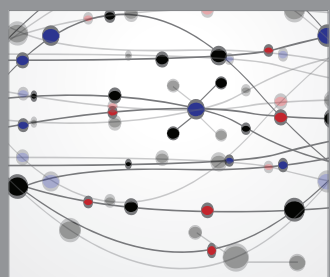

The Scientific World Journal
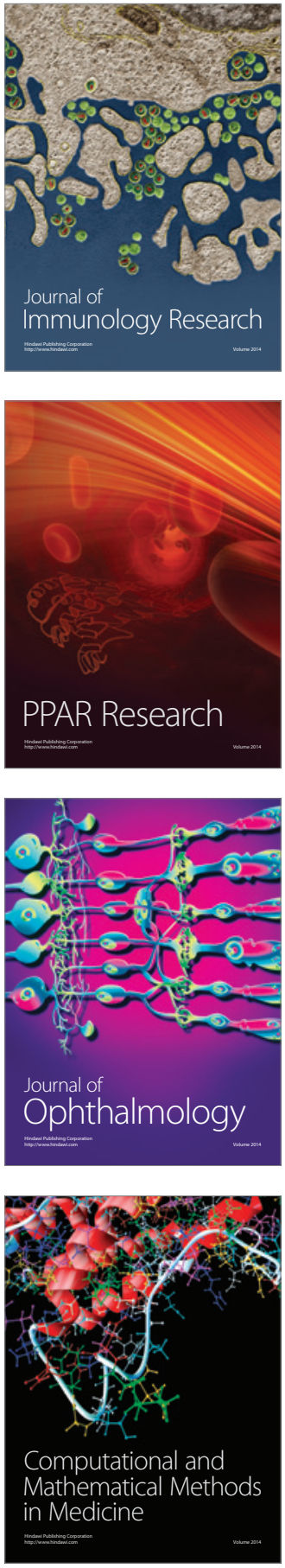

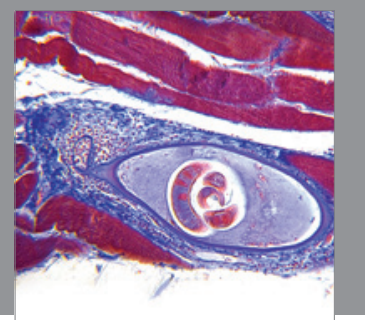

Gastroenterology

Research and Practice
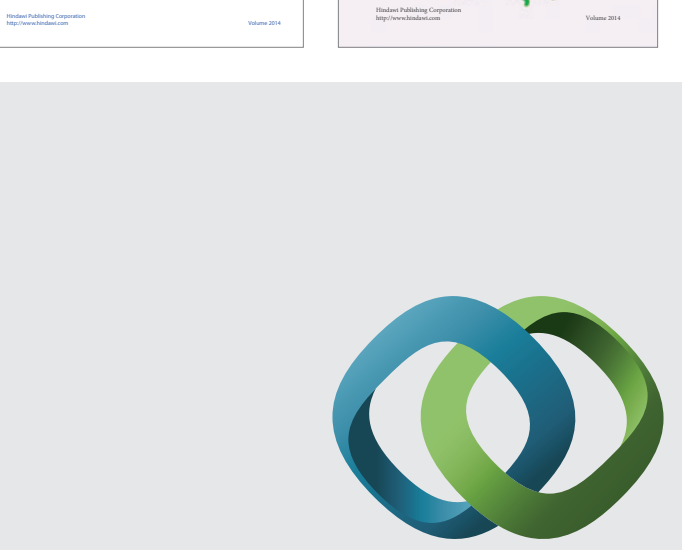

\section{Hindawi}

Submit your manuscripts at

http://www.hindawi.com
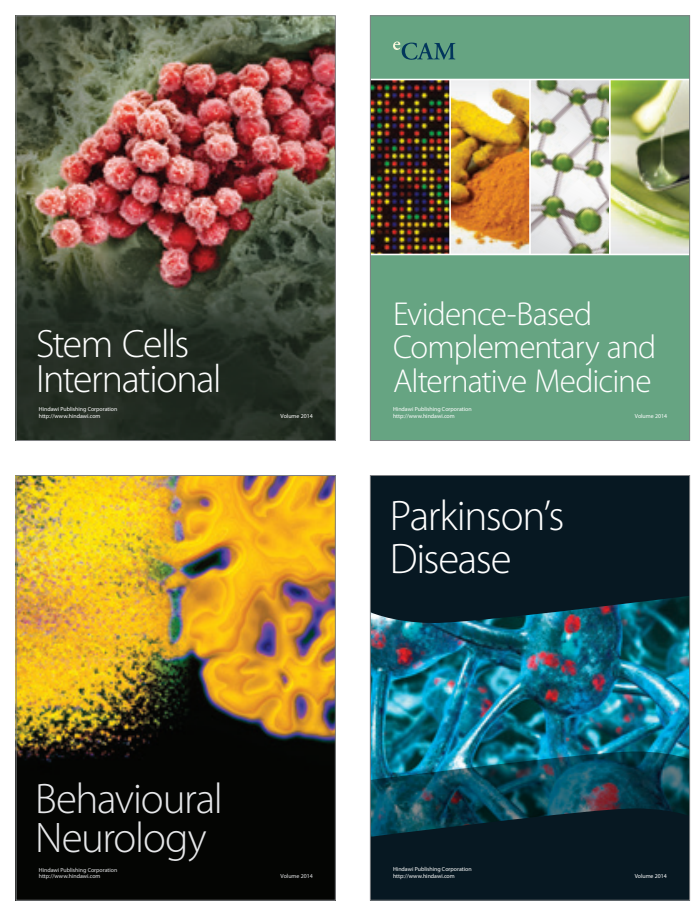

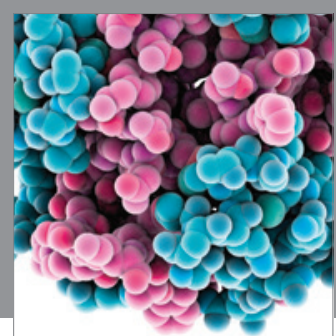

Journal of
Diabetes Research

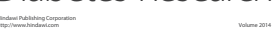

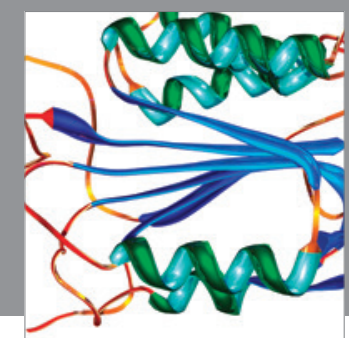

Disease Markers
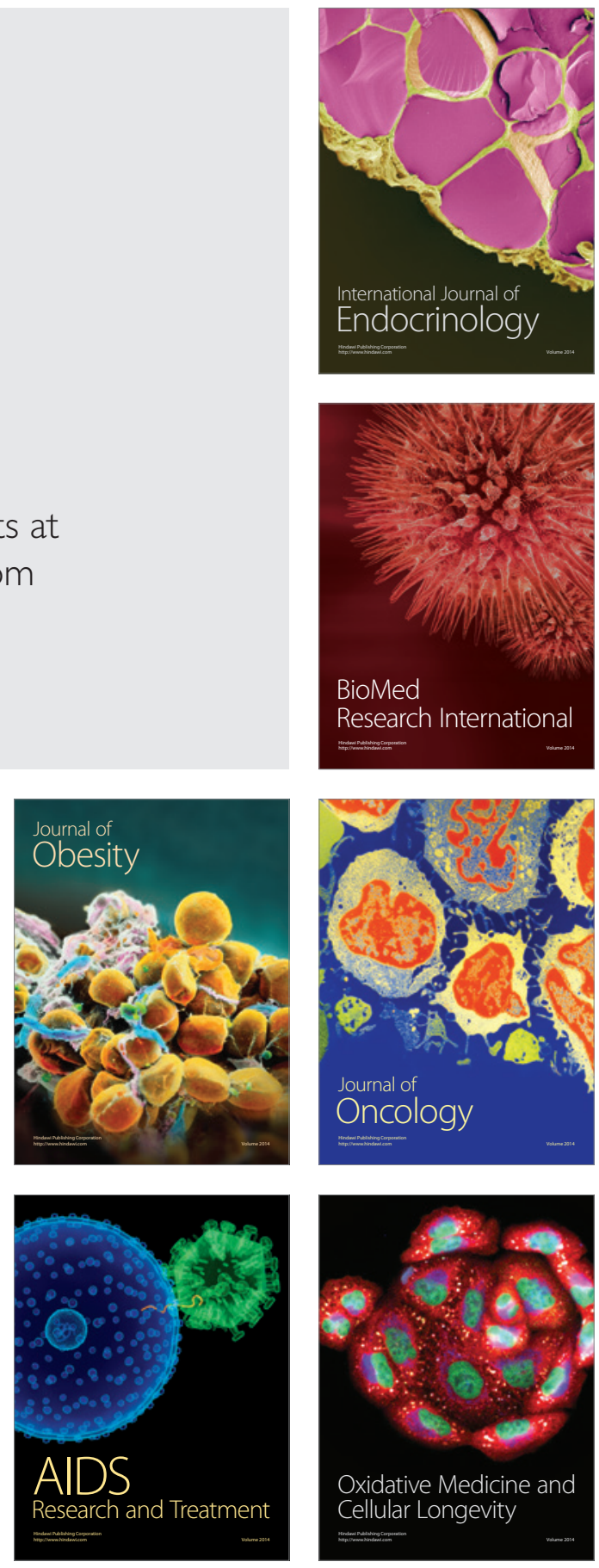